\title{
PATTERNS FOR IDENTIFYING APPROPRIATE KNOWLEDGE MANAGEMENT STRATEGIES IN ORGANIZATIONS
}

\author{
Khadijeh Salmani \\ Department of Executive management, Science and Research Branch, Islamic Azad University, Ardabil, Iran.
}

\begin{abstract}
Today, striving to find an efficient way for developing or identifying appropriate knowledge management strategies in organizations has become so critical that has attached information pyramids in organizations a great importance, and has highlighted lack of trained and skilled staff as a serious problem. On the other hand, having more flexibility at workplace, offering better service and fulfilling customers' demands require a strategy for managing knowledge and its consequences. Knowledge management enables a wide range of different strategies and methods for identifying, creating and sharing knowledge in organizations. This deep insight includes individuals and organizations' experience and knowledge and understanding it helps the organization respond to both internal and external stimuli and act in harmony. One fact which has seemingly achieved consensus is the need for different strategies of knowledge management. Among the wide range of various and often unclear knowledge management strategies one can choose a strategy in a specific situation. The aim of knowledge strategy is to respond to strategic questions which emphasize competitive intelligence and internal knowledge retrieval systems.
\end{abstract}

Key Words: knowledge management, knowledge classification, pattern, strategy.

\section{Introduction}

In order to use the knowledge asset in organizations more aptly, a field called "knowledge management" was created. In other words, the area of knowledge management (KM) was founded on the basis of believing in extending and further using knowledge properties (or intellectual capitals (IC)) to attain competitive advantage (Marr \& Schiuma 2001). Knowledge management (KM) or sharing knowledge is in fact creating, understanding and spreading knowledge in organizations. Putting knowledge management into practice is 
actually an effort to benefit from the knowledge through which persons in organizations can accomplish the organization's mission (Mc. Inerne 2002). Erickson believes a company can reduce its rivals through using special knowledge, information or data and can hence better prognosticate anti-competitive measures (Andreou \& Bontis 2007).

HCG (Harvard Computing Group 1998) holds that knowledge management systems let the organization store, diffuse and utilize others' information and experience, and that the aim of knowledge synthesis is improving business activities. It can be said that in modern knowledge age the organization will not survive unless it applies a strategy to exert influence on its properties. Plenty of strategies are suggested in knowledge management cycle. The term "knowledge management" is designed and used for creating and increasing intellectual properties and exchanging them within organization in a wide variety of activities. In fact, it is the very knowledge management on which consensus has been reached. Knowledge management can play an important role as an ingenious plan for the processes, instruments, structures and etc. with the purpose of reviving/renewal and sharing knowledge through improving the use of intellectual capitals of each structural, human and social elements (Seemann et al. 1999).

\section{Knowledge Classification Based on Nunaka and Takuchi's Matrix}

Classifying knowledge in optimum ways is a controversial issue (Beckman1999). Some classifications that can be useful are based on a synthesis of knowledge accessibility (the place where knowledge is stored and the form in which it is stored) and evolution (the flow of knowledge from one place to another and from one form to another). This viewpoint set the scene for the spiral analysis and interpretation of knowledge by Nunaka and Takuchi. It also occurs as an information model ("I-Space") of Boisot's extended model in course of knowledge reform in an innovation and learning... (Nunaka \& Takuchi, 1995). A widely accepted approach to knowledge classification is knowledge matrix from the viewpoint of Nonaka and Taekuchi. This classified knowledge matrix is either explicit and implicit, or individual and team-based. Nunaka and Takuchi interpreted the processes of the transformation of knowledge from one form to another as:

- Getting socialized (implicit to implicit): makes an individual get the implicit knowledge either directly from others or through shared experiences, observation and imitation.

- Explicit suggestion (implicit to explicit): through expressing implicit knowledge in explicit and clear concepts.

- Synthesis (explicit to explicit): through cooperation of a set of concepts while considering the different inputs of explicit knowledge.

- Centrality (explicit to implicit): through a process of learning and performing it or through observations, experiences and documents.

In the process of creating organizational knowledge Nonaka and Takeuchi's model acts as a space wherein knowledge is strengthened in four ways. The knowledge is enhanced within the organization and on levels higher than individuals or groups inside the organization and even moves within the organization. 


\section{Second Classification of Knowledge: Boisot's I-Space}

Boisot (1998) developed the extended model of knowledge property in an effort to complement Nonaka and Takeuchi's theory, but the Boisot's model introduces some other dimensions (Boisot, 1998). For instance, in his model, "abstraction" means that we can generalize knowledge to different situations. Such classification allows for a more detailed flow, transfer, and analysis. In Boisot's model, one can define knowledge supply in a threedimensional space from uncodified to codified, from concrete and absolute to abstract, and from undiffused to diffused. He then uses Social Learning Cycle (SLC) in the space of knowledge flow model which is comprised of six stages:

1. Scanning: spreading available data (diffusion)

2. Problem-solving: solving problems of structure and giving coherence to it (knowledge is codified).

3. Abstraction: the new insight is generalized to a wide range (the knowledge becomes more abstract).

4. Diffusion: the new insight toward the target community is shared in an abstract and codified way (knowledge is propagated).

5. Absorption: the new or newly-codified insight is used in new experiences of learning process (the absorbed knowledge leads to some new behaviors and in fact becomes tacit or implicit).

6. Impacting: the abstract knowledge is lodged in concrete behaviors. For example, the principles and behavioral patterns take ancient form (knowledge becomes concrete).

In Boisot model, interesting programs develop from thermodynamic rules, i.e. the knowledge supply is very abstract, codified and undiffusible.

\section{I-Space Model Knowledge Properties (the minimum level of abstraction, codification and diffusion)}

This thermodynamic comparison alludes to the elusive and dynamic nature of knowledge. It seems that something is happening in this cycle and the data are filtered for the production of meaningful information. And then this abstract and coherent information can help with production of knowledge. As knowledge is used in different situations, the new experiences enter the new cycle of knowledge creation in the form of undiffused experience as well as produced data. It can be concluded that in both models, i.e. Boisot's and Nonaka and Takeuchi's, the properties of knowledge in organizations are always changing and follow an upward process. Organizations are live beings that should always adjust themselves to their environment. It means, knowledge management strategies should always progress in the appropriate and determined time and should improve their knowledge, i.e. should enter the new stage through organizational learning cycle. The amount and rate of change in this cycle vary from one part to another In a way that the rate of completion of new knowledge may be high in one part while in some other parts it is really low. 


\section{Classification Based on Business Process: an Investigation of Determining International Barter Criteria (APQC)}

Karl Wiig and APQC (American Productivity and Quality Center) have considered six strategies for knowledge management that reflect the essence, strengths and relevant methods of involved organizations (Wiig 1997\& Manasco 1996).

- Knowledge strategy as business strategy: is a large-scale investment approach where knowledge is often considered as a product.

- Intellectual asset management strategy: puts emphasis on within-company assets that may be completely abused.

- Strategy of personal knowledge asset responsibility: encouragement and support for every single employee to extend his/her knowledge skills and share their knowledge asset with others.

- Strategy of knowledge creation: accentuates innovation and creation of knowledge through the system of research and development. The way it can contribute to prospect through determining and shaping the routes is consented by market leaders.

- Strategy of knowledge transfer: knowledge transfer is the best way to improve quality and operational profitability.

- Focus on customer knowledge strategy: with the aim of understanding and exactly responding to the very needs of customers.

\section{Binney's Spectrum of Knowledge Management Approaches}

Nickols (2000) suggests that Nonaka and Takeuchi should have focused on declarative knowledge or its procedures. In the classification of knowledge management approaches spectrum it is suggested that this spectrum can complement the different methods discussed in previous sections sufficiently, thoroughly and usefully. In a framework, Binney (2001) presented the spectrum of knowledge management under the title of knowledge management instruments for helping the organization and evaluation of situations in which knowledge management terms are used.

His emphasis on knowledge management activities is classified into six categories:

- Transactional Knowledge management: knowledge is included in technology. Analytical knowledge management: knowledge is derived from external information sources, and normally, its focus is on information related to customers.

- Asset management of knowledge management: Managing the asset of knowledge (which is often created as a by-product of business) is explicit and can be recycled in a variety of ways.

- Process Knowledge management based on business: codification and improvement of business policies and sharing the improved policies within the organization.

- Developmental knowledge management: developing ability and knowledge in organization members through training the staff. 
- Innovation/ creation knowledge management: improving the environment and creating new knowledge for example through $\mathrm{R} \& \mathrm{D}$ and via forming teams in different fields.

\section{Knowledge Management Background}

Plenty of investigations in the area of knowledge management are carried out by different researchers and published in different journals, but Davenport and Cronin (2000) have described knowledge management commitment in three areas: a) librarian and scientific information (KM1- information management is another name for knowledge management). b) Process engineering (KM2- business process, ontology and managing the way of knowledge formation). c) Organizational theory (KM3-knowledge as a source and ability to turn implicit knowledge into explicit within the framework and atmosphere of organization). The purpose of presenting these three frameworks of knowledge management is to provide a means for analysis and interpretation and study of tensions that might exist in the organizations committed to knowledge management. There are different interpretations of knowledge management in different areas. The results indicate that there are different changes in these three areas of knowledge management. Knowledge management is not information management. However, information management is a subset of knowledge management and thus includes a variety of ways in which knowledge can be used within the organization's boundaries.

\section{Knowing two Important Approaches in Knowledge Management Strategy}

According to Wiig (1997) knowledge management is an explicit and systematic process for creation, renewal and activation created for the purposes of magnifying and restoring basic and useful knowledge. Different researches are conducted on proposing typology for management strategies which can reflect different knowledge management processs (Wiig,1997). Through studying professional companies and relationships between management and Hansen et al.'s (1999) valuable information, two major strategies of companies are characterized: codifying approaches and personalizing knowledge management. The difference between these two strategies arises from their tendency toward knowledge. One believes in the concreteness and separation of knowledge from human forces, but the other one does not believe in the significance of such segregation. The main concept of knowledge formation comes from management debates. These two major knowledge management strategies are not specific to one profession or industry (Wiig 1997\& Hansen et al. 1999).

\section{tCodification Approach}

In knowledge management codification approach an electronic system encodes and stores a document and allows for its diffusion and reuse. Competitive strategies, for those components which are suitable for codification approach, can be specified by commodity strategy. The commodity strategy places great importance on economical aspect of reuse.

Initial investment in extracting, codifying and reporting knowledge for important processes and applying knowledge to resolve many of analogous issues ultimately lead to knowledge in large scales. Many users utilize knowledge without having contact with the major source of expertise. In order for knowledge codification strategies to succeed, the processs which knowledge of a company looks for managing are identified with their all 
concomitant internal and critical profits for business. Knowledge management includes great amounts of energy and organizational resources, and due to lack of diagnosis it cannot provide background for anything. In other words, these two approaches come from two different fundamental knowledge theories. Codification approach expresses awareness of an external issue that can be transferred to experts while in personalization approach it is observed that this awareness is separable from the quality of the experts. Each of these observations reflects a different concept of knowledge management trends. Stratification strategy which is codification approach of knowledge strategy is for supplying and reuse of large quantities of library knowledge. While personalization approach focuses on the facilitation of using experts for improving the discussions. These two approaches to knowledge management are more comparable with issues such as strategy for the inclusion of knowledge management, information technology requirements, employment of human resources and policy setting. An organization's decision in choosing major knowledge management approaches is a function of organization's business core, meeting the needs: advanced technology, crisis trends and organization's culture. The knowledge management strategy chosen by the company should include its business strategy.

\section{Personalization Approach}

Facilitating the improvement of information and personal communication systems is the core of knowledge management approach in organizations. Therefore, organizations can change their ideas and properties through communication and socialization. For companies interested in codification approach a new strategy can be determined. A new strategy approves economical instead of repeated uses of the same expert. The issue of customer is not an example of a generic issue, and in finding a generic solution to a problem the purpose of newly-founded strategy is not to invest once and use several times. Instead, the company usually uses small cohesive expert groups. So, personalization strategy focuses on experts and the thought of newly-founded human resources.

Developing basic relationship for facilitating communications and changing ideas among human resource experts is the main issue in information systems dependent on knowledge management. Since we still need human expertise to access necessary information and data for processing information, it is not clear yet how knowledge can be codified and stored and how these information and data can be accessible for retrieval and reuse to either human or automatic system users. Selecting and implementing a chosen approach is not always a direct, straightforward duty. Managers' competition for selecting appropriate codification and personalization approaches is accompanied by careful evaluation of the conditions, and this prevents from undergoing other processes. These processes come from several sources, including: the complexity of the domain of the problem and a professional technical coverage which is limited to non-technical staff. Put differently, to select and implement knowledge management successfully we need to identify and spread it (Wong \& Tiainen, 2004).

\section{Knowledge Management Strategy: Models, Designs and Concepts}

- Neto (2005) believes that knowledge management system is written as a unified concept. Three frameworks support his viewpoint:

- The strategic conceptualization of knowledge and information; Suggested by Choo (1998) as a competitive factor for society and organization. 
- The concept of creating an atmosphere of tactical or strategic knowledge in organizations or situations for using the best information available in the organizations proposed by Wong et al.

- Introducing a strategy at an operational level through different management methods and information technology tools sensitive to relationships, called "knowledge management umbrella". This knowledge management umbrella used as a metaphor for ideas, thoughts and methods of management and IT, is used within the organization in many areas as a tool related to knowledge and information. It is essential that issues such as "management of strategic information", "competitive intelligence", "organizational learning" and intellectual capitals... to be highlighted. This is the same conflict among teams which brings about creation of a theoretical framework designated as "knowledge management".

\section{Strategic Meaning of Knowledge and Information in Organizations}

According to Choo (1998) organization's knowledge is in the hands of those who use organization's information strategically in three stages: a) sense-making b) creating knowledge, and c) decision-making.

- Sense-making: the objective is to be able to let organization members have a mutual and shared understanding of whatever goes on in the organization. Strategy is more reflected in organization's mission, prospect, values and culture. It provides the members with opportunity to visualize the meaning of their work and life and have an ambitious and challenging prospect of future. Communicating, using knowledge and improving it are of high importance in making sense of organization's goals. The new objectives are adjusted to the pre-determined goals of the organization in the long run, and through changing the information and prospective activities create dynamism and prosperity in the organization and let the changes, methods, procedures, customers' scenarios, suppliers, competitors and other persons and the external environment to be understood. Organizations continually face difficulties such as increase of uncertainty and complicated dimensions of management. Organizational designs which receive no clear answer involve competitors, competitors' social intelligence, environment, scientific researches in the market and similar activities.

- Creating knowledge: it is a process which lets the organization obtain and create improved or organized information through learning in order to create new knowledge. The new knowledge in itself helps the organization extend new abilities and capabilities, offer new service and products improve and design repeated processes for organization. Creating organizational knowledge is in fact strengthening knowledge by people based on the conditions that should be offered or activated in organization. In this regard, knowledge management actually means visualizing the prospect as well as a new interpretation of knowledge as the source of abilities in managing knowledge.

- Decision-making: in Choo's model, the company opts for the best among the acceptable choices, and those that are closer to the objectives and strategy of organization. According to rationality principle of Mars and Simon (1975) decisionmaking process is a limited action. It is inferred from the theory of Choo, Mars and 
Simon that in some decision-makings, in quest for an optimal solution, an alternative satisfactory, and apt enough solution can be arrived at (Simon1975).

\section{Making Connections between the Two Strategies}

According to King (2001) knowledge management strategies have close relationship with organizational learning strategies. Through amalgamating their concepts, it can be said that organizational learning is attained by knowledge management strategies. King helps understand the issue that knowledge management strategies induce an increase in organizational learning. On the other hand, it is said that senior manager's strong support for strategy leads to an increase in organizational learning and reinforcement of knowledge management strategy. Cohen (1997) in a report states that top managers' support has great effects on strengthening internal culture of an organization, and in the quest for attaining the purposes and values of the organization, it can share perspectives through creating the best situation.

\section{Some strategies required in knowledge management approach}

1. Senior management's support: as mentioned before, for optimal implementation of the top knowledge management strategy, knowledge management support is needed. Senior managers should be involved in the exchange of implicit knowledge between the communities of knowledge. Through consistently supporting peer groups in organization and rewarding them, they can assist in sharing the information all over the organization. Through interventions in such processs senior managers actually facilitate the creation of common values within the organization. They trigger production of new ideas and increase of valuable intellectual assets in organization by creating an atmosphere wherein normal business processes consistently improve.

2. Common values: common values arising from organizational culture are important for increasing organizational knowledge in the form of knowledge management strategies. To increase organizational learning, the right structure makes a set of common values for developing effective knowledge management strategies. Senior managers should reward change agents in the organization and those staff who regularly take part in sharing knowledge and promoting organizational learning.

3. Peer groups: peer groups should play some role in sharing knowledge in organizations. One important feature of the organization's knowledge management is that organizational learning should be established and promoted through building relationships and group communications. Management should be cognizant of knowledge hoard by those staff that have key information, and through encouragement and rewards persuade them to impart their knowledge to the whole organization.

4. Quality management and constant process improvement: quality management strategies ISO9000 are powerful tools for knowledge management as a knowledge strategy which is specially a transformer of tacit knowledge into codified knowledge. ISO9000 standard methods are carefully performed by staff in their routine tasks. When methods of a process are well-documented, the organization should study systematic processes and improvement of daily activities in order to identify the opportunities. Process improvement includes consistently studying existing processes and trying to diagnose new and better ways for managing business. 
5. Innovation: after common values, peer groups, quality management and consistent improvement of process, innovation can be a bridge for knowledge management strategy. Innovation in knowledge management strategy is of paramount value. The new idea created by the staff should be valued by the organization. Expressing and sharing ideas within the organization should be part of the organizational culture. Peer groups should also be allowed to express and share new implicit knowledge with other members and be assured that their new and brilliant ideas will be implemented. Consistent improvement of processes regularly encourages organization members to create new ideas and value for the organization. Members whose ideas are of value to the organization should be rewarded.

\section{Strategy of Knowledge diffusion Mechanism}

The extent, to which knowledge can be transferred to other organizations voluntarily or involuntarily, through overflowing, depends on the nature of the knowledge used. Explicit or structured knowledge is simpler to use and is likely to become available to the competitors (Boisot, 1998). Therefore, the best thing to do is to use structured knowledge rather than diminishing the value of that knowledge by transferring it to the rivals without supervision. The strategy of blocking knowledge by legal means such as registering innovations, copyright for or concealing knowledge however, can somewhat prevent from knowledge diffusion to competitors. But more profits can be attained in the long run through choosing sharing strategy in environment (Boisot, 2003). In studying organizational behavior, to select in a strategic way the mechanism of guiding evolution in a group of organization should be considered by each single company. In a simulated model we can examine how some knowledge management strategies can be selected and a group of companies or organizations can be affected.

Knowledge management strategies are very complicated and diverse, and due to social complexities and experiential problems, the features of the real world cannot be incorporated into a simulated model. And undoubtedly the simulated model is always simpler than existing realities. In this simple example there is an attempt to show the key features of the study as much as possible. Therefore, only two dimensions of company's knowledge management strategies about people's behavior are analyzed.

6. First stage: Structuring knowledge based on factors applied in it. Structured knowledge has an easy process of transfer in database and documents conditioned that the code and abstract designs of knowledge take on a common structure.

7. Second stage: Transfer of feelings or values. Face-to-face interaction usually needs a wider bandwidth which is related to all non-verbal communication methods.

\section{Conclusion}

Structured knowledge can be easily used and shared. Leakage of knowledge is easy, but extracting its value is difficult. Totally speaking, the probability that knowledge can be considered as asset depends on its level of diffusion. It means, to some extent, companies and organizations codify and apply both structured and unstructured knowledge. Companies can use structured or less structured strategic knowledge based on their priorities. Without question, companies, with their different abilities and capacities, can find knowledge asset in vaying degrees. The second knowledge management strategy is blocking its diffusion. 
enterprises or companies can control the knowledge as a free flow and without any obstacles to their own benefit through adopting a strategy with the aim of blocking knowledge via tools such as registering invention, creating patent law/copyright or concealing and hence influence the market.

\section{References}

Alvarenga, Neto R. (2005), "Gestão do conhecimento em organizações: proposta de mapeamento conceitual integrative", Doctoral thesis in Information Science. Belo Horizonte: PPGCI, Escola de Ciência da Informação da UFMG.

Andreou, A. and Bontis, N. (2007) , "A Model for Resource Allocation Using Operational Knowledge Assets," The Learning Organization, Vol. 14, No. 4, pp 345-374.

Beckman T.J., (1999), “The Current State of Knowledge Management, in the Knowledge Management Handbook”, ed. J. Liebowitz, CRC Press.

Binney D.(2001). "The knowledge management spectrum-understanding the KM landscape, Journal of Knowledge Management", 5, 1, 33-42.

Boisot M.H.(1998)., "Knowledge Assets: Securing Competitive Advantage in the Information Economy", Oxford University Press.

Boisot, M.H. and Canals, A.(2003). "Modeling knowledge-based economic processes: A simulation approach". Paper presented at OKLC 2003, April 13-14, 2003, Barcelona.

Choo, C. (1998). "The knowing organization: how organizations use information for construct meaning", create Cohen, Don. Managing Knowledge for Business Success. The Conference Board, 1997, Report Number 1194-97-CH.

Davenport, E. and Cronin, B.(2000). "Knowledge management: semantic drift or conceptual shift?" Journal of Education for Library and Information Science, 41 (4), 294-306.

Hansen, M. T, Nohria, N. and Tierney, T.(1999). "What's Your Strategy for Managing Knowledge?", Harvard Business Review(March-April): 106-116

Harvard Computing Group, (1998),"Knowledge Management and Return on Investment”, Vol.1, N.2

King, R. (2001), "Strategies For Creating A Learning Organization", Information Systems Management, 2001, Vol. 18, Iss. 1, pp.12- 20.

Kruger, E.(2007), "Formulation of a Strategic Knowledge management maturity model, Internet. knowledge and make decisions". New York: Oxford Press.

March, J.and Simon, H.(1975). "Limites cognitivos da racionalidade". Teoria das organizações. Rio de Janeiro:Fundação Getúlio Vargas.

Marr, B. and Schiuma, G. (2001) "Measuring and Managing Intellectual Capital and Knowledge Assets in New Economy Organisations", in Bourne, M. (Ed.), Handbook of Performance Measurement, Gee, London.

McInerne, c. (2000), "knowledge management the dynamic Nature of knowledge", journal of the American Society for Information Science and Technology, 53(12):1009-1018,

Nickols ,F. ( 2000), “The Knowledge in Knowledge Management”, The Knowledge Management Yearbook 20002001, ed. J.W. Cortada and J.A. Woods, Butterworth-Heinemann, 12-21.

Nonaka I. and H. Takeuchi, (1995), "The Knowledge-Creating Company: How Japanese Companies Create the Dynamics of Innovation", Oxford University Press.

Nonaka, I. (1991). "The knowledge creating company”, Harvard Business Review 69 (6 Nov-Dec): 96-104.

Seemann P., D. De Long, S. Stucky and E. Guthrie, (1999), "Building Intangible Assets: A Strategic Framework for Investing in Intellectual Capital", Second International Conference on the Practical Applications of Knowledge Management (PAKeM99), 21-23 April.

Wiig, K.(1997). “Knowledge Management: Where Did It Come From and Where Will It Go?”, Expert Systems with Applications, 13, 1, 1-14

Wiig, K. (1997). "Knowledge Management: Where Did It Come From and Where Will It Go", Journal of Expert Systems with Applications 1997 (Fall). 
Khadijeh Salmani, International Journal of Organizational Leadership, Vol. 1 No. 1 (2012)

wong, R. and Tiainen, T. (2004)."Strategic knowledge management: In search of a knowledge-based organizational model", Comportamento Organizacional E Gestão, 2008, VOL. 14, N. ${ }^{\circ}$ 2, 247-256. 